\title{
PELVIC OSTEOTOMY IN THE TREATMENT OF ECTOPIA VESICAE
}

\author{
G. C. Lloyd-Roberts, D. I. Williams and G. T. F. Braddock, London, England \\ From the Hospital for Sick Children, Great Ormond Street, Lomdon
}

This is a preliminary report on a method of pelvic osteotomy which we have found of value in the treatment of ectopia vesicae.

The operation involves complete division of both iliac bones from crest to greater sciatic notch, together with the sacro-tuberous ligaments. A few days later fibrous tissue is removed from the pubic symphysis, the bladder is buried, and the divarication of the recti and the skin defect are repaired. There is only moderate tension on the suture lines because the inward rotation of both sides of the pelvis which is possible after iliac osteotomy enables the pubic rami to be approximated (Figs. 1 and 2).

\section{SOME ASPECTS OF THE ANATOMY AND THE SURGERY OF ECTOPIA VESICAE}

Ectopia vesicae is a complex malformation in which there are two basic defects. First, there is a failure of midline union of the anterior wall of the bladder, urethra, pelvic ring and attached muscles and of the anterior abdominal wall. The lower urinary tract is therefore laid open upon the surface of the lower abdomen, protruding through a triangular gap between the diverging recti abdominis. The os innominatum is rotated laterally upon the sacrum so that the pubic bones, separated in the midline, form two prominences in the groin outside the bladder area. The pelvic ring is, nevertheless, stable, because the pubes are united by strong fibromuscular bands incorporated in the base of the bladder. In epispadias (a lesser degree of the same deformity involving only the bladder neck and urethra) the pubes, although separated, are united by a band of fibrous tissue which lies in front of the bladder, thus allowing better development of the sphincter.

The second defect is shortening in the sagittal plane. The umbilicus is drawn downwards so that its scar is usually blended with the apex of the bladder. The whole urethra is shortened, and in the male it is drawn upwards and forwards to lie dorsal to the corpora cavernosa. The verumontanum lies closely below the trigone area, the sphincteric region is short and ill developed, and the penis is stumpy and upturned. The anus is drawn forwards in the perineum and is often poorly controlled. In the female the urethral strip is short and wide, the vaginal orifice is directed forwards immediately behind it, with the bifurcated clitoris on either side.

The skeletal defect causes curiously little disability. The limb is somewhat laterally rotated, and the gait, although waddling in childhood, is hardly distinguishable from normal in the adult. In contrast the continual and unmanageable incontinence associated with ectopia vesicae is a challenge to every urological surgeon, who in despair often performs a urinary diversion, which is so often followed by its own formidable complications. The ectopic bladder provides unpromising material for constructive surgery, but in many cases there appears to be good muscular tissue which might serve in a functional reservoir if it could be properly disposed. Numerous attempts have been made to attain continence, but in very few has even limited success been achieved - and then usually only in girls.

Such muscle as remains would be expected to act as a more competent sphincter if it were not tightly stretched between widely separated pubic bones, thus closing the pelvic ring, a task properly performed by the skeletal system. A direct union of the pubic bones is therefore a desirable preliminary to bladder construction, and it is with this problem that we are here concerned. 


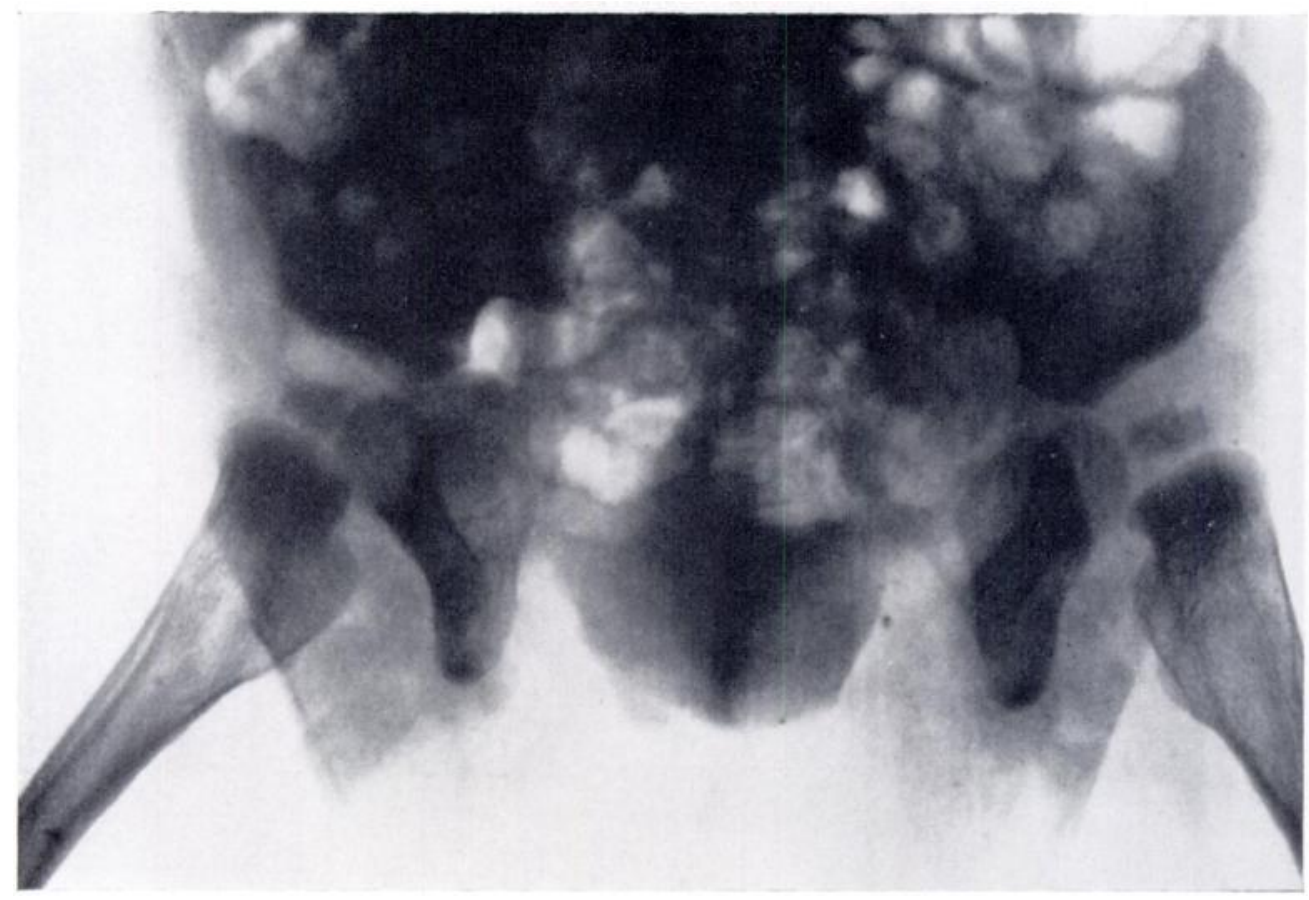

FIG. 1

Radiograph to shcw the extent of the pubic separation before pelvic osteotomies.

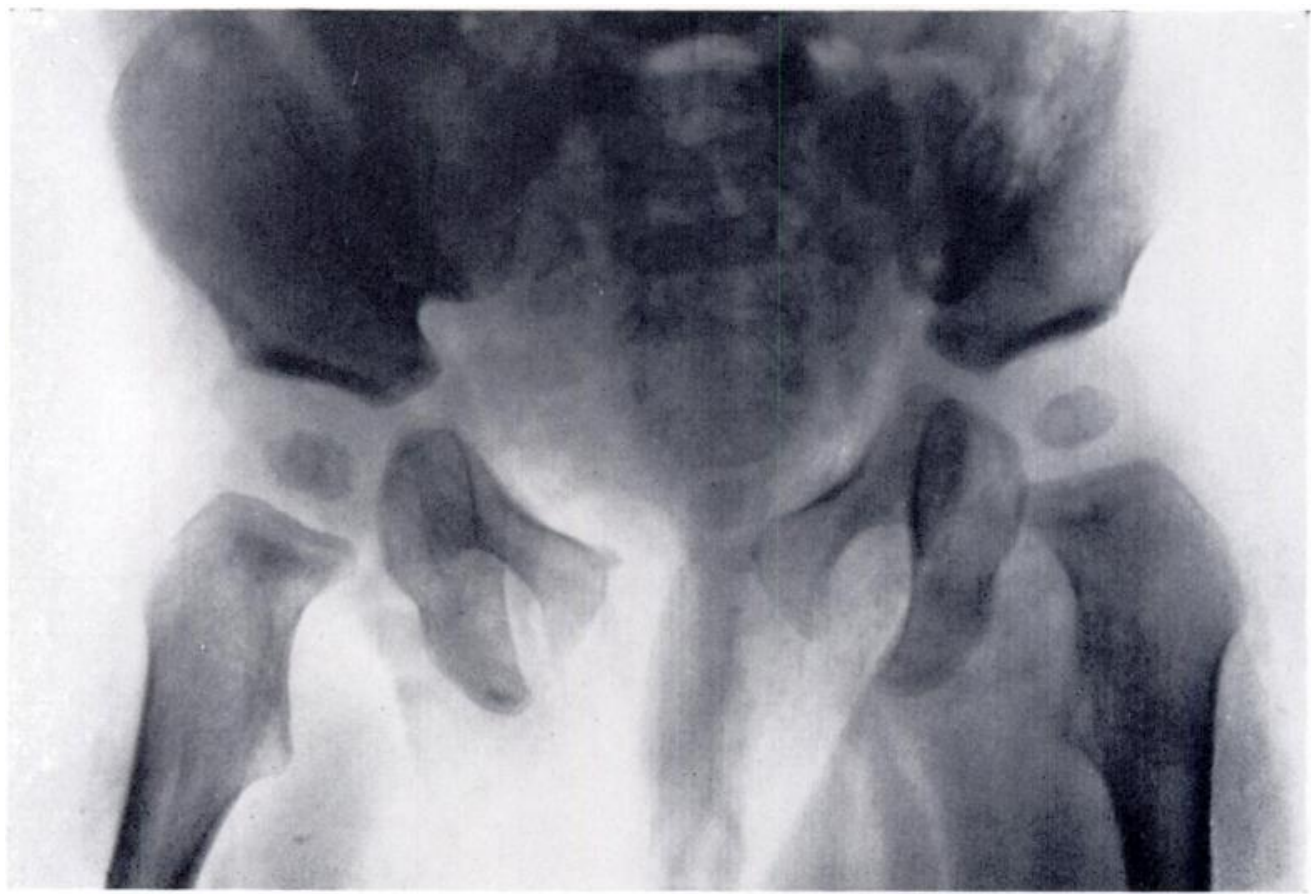

FIG. 2

Radiograph of the same patient after pelvic osteotomies and subsequent closure of the bladder. The position of the osteotomies on the ilia is shown. The extent to which they have opened to allow approximation of the symphysis pubis can be seen.

VOL. 41 B, NO. 4, NOVEMBER 1959 


\section{THE OPERATION}

The baby lies prone and an intravenous infusion is advisable. The operation is performed through two incisions, one for each side. The incision is marked on the skin and runs along the posterior half of the iliac crest, curving downwards in the line of the sacro-iliac joint as far as the posterior inferior iliac spine. The area of operation on each side is infiltrated with about 15 millilitres of a solution of 1 in 200,000 adrenalin mixed with hyalase. The incision is deepened through the deep fat until the cartilaginous iliac epiphysis is seen. Thereafter it continues downwards until the junction of the sacrospinalis and the origin of gluteus maximus is clearly displayed. The plane between these muscles is developed and the iliac bone exposed subperiosteally. Subperiostal stripping is continued until the posterior surface of the ilium is widely exposed from the iliac crest to the greater sciatic notch, around

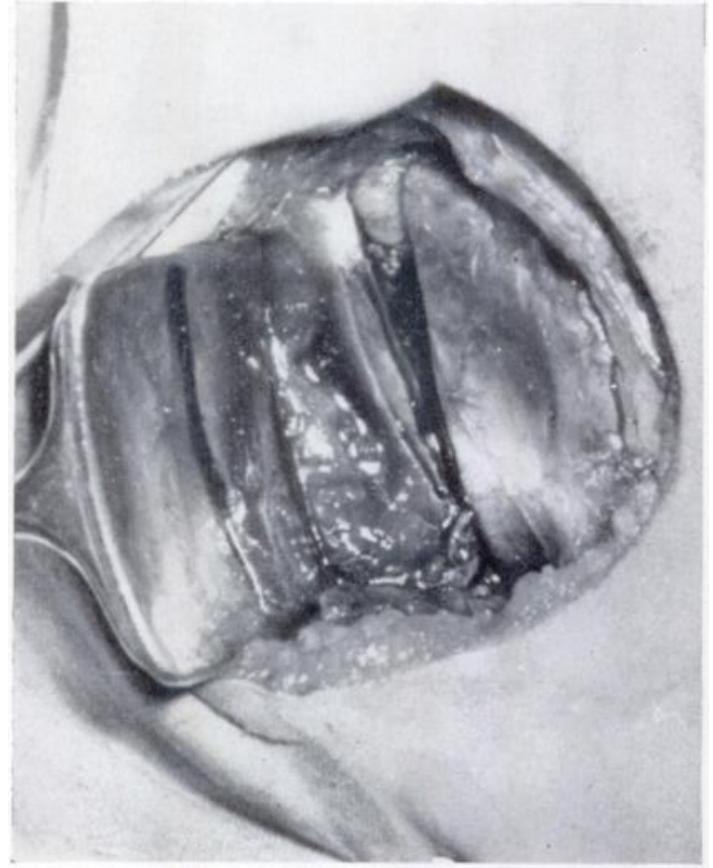

FI(i. 3

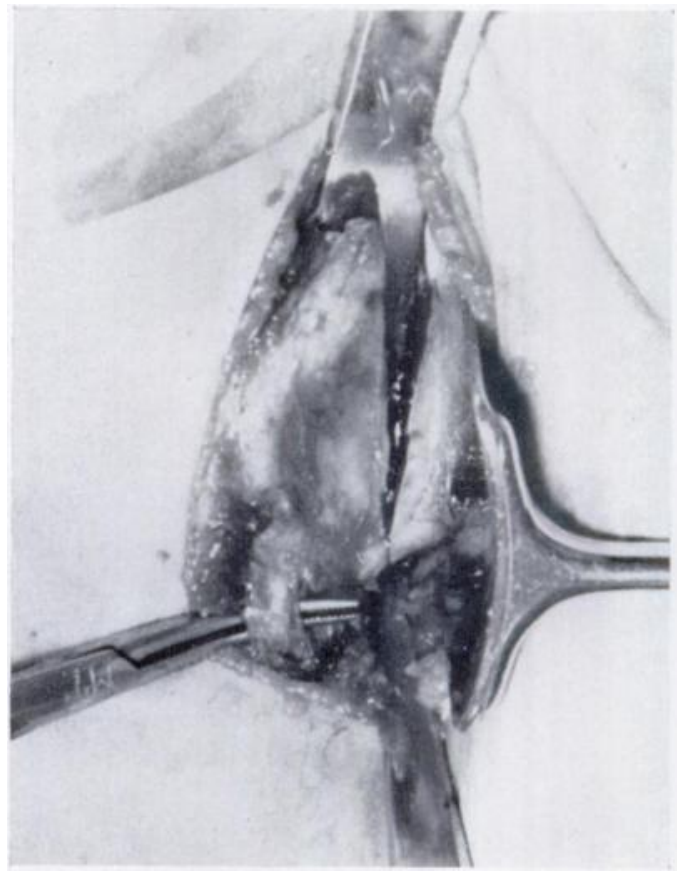

FIG. 4

Figure 3-Left side. The osteotomy has been completed and the pelvis is being compressed to confirm this. The sacral attachment of the sacro-tuberous ligament can be seen in the lower right corner of the wound. Figure 4-Right side. The sacro-tuberous ligament is isolated over an artery forceps. The lower bone spike enters the pelvis through the greater sciatic foramen.

which a bone spike is placed with its point lying within the pelvis. In our early cases some anxiety was felt lest the superior gluteal artery be injured and retract within the pelvis: but these fears have not been realised.

Attention is now turned to the iliac crest, from which the posterior abdominal muscles are dissected until a finger can be passed around the pelvic surface of the ilium. The osteotomy is performed from above downwards, the intrapelvic finger defining the upper limit and the bone spike the lower limit, where the osteotomy must be carefully completed under direct vision lest the inner cortex be left undivided. Compression of the pelvis both confirms that the osteotomy is complete and also tenses the sacro-tuberous ligament, which can be felt as a cord stretched between its fanlike attachments (Fig. 3). A blunt hook is passed around the ligament, which is divided (Fig. 4). The muscles, fat and skin are sutured individually and the operation is repeated on the other side of the pelvis. 
After the operation the baby is supported by "gallows" traction, which both protects the wounds from contamination and draws the two halves of the pelvis together.

The second stage - closure of the bladder-is usually performed three or four days later and the "gallows" traction is maintained until the abdominal wound is healed.

\section{DISCUSSION}

We have carried out this operation seven times. Six patients had ectopic bladders and one patient, a child of three, had an exceptionally difficult epispadias. In this latter patient the operation failed to simplify the second stage of plastic repair. We believe that the age of the child was the important factor in this, and we now prefer to operate before the age of one year and preferably at six months or earlier. Our colleague, $\mathrm{Mr} \mathrm{H}$. H. Nixon, has twice closed the bladder successfully in newborn babies after manual osteoclasis of the pelvis. In both it was possible to repair the abdominal wall. Nixon (1957) and Schultz (1958) were, as far as we can determine, the first to apply this principle in the treatment of ectopia vesicae.

Five out of the six patients in whom this osteotomy has been performed have had their bladders closed primarily without fistula formation. In the last four-in whom division of the sacro-tuberous ligaments has been added to the osteotomy-it has been possible to repair the recti. which both restores the normal structure of the abdominal wall and provides a substantial muscular layer between the bladder and the surface. In one patient (our first) the wound broke down and the condition recurred two months after our apparently successful closure. In this instance the sacro-tuberous ligaments were not divided, and it is probable that the osteotomy was incomplete at the lower end.

Closure of the bladder is, as we have already emphasised, only the first stage in the possible development of an operation which will enable sphincter control to be restored. However, bladder closure alone has certain obvious advantages. Cosmetically the removal of so offensive a deformity and the cause of urinary excoriation of the abdominal wall is clearly desirable. Furthermore, successful repair of the separated recti should prevent the development of a large ventral hernia. In girls the restoration of the integrity of the abdominal wall is an advantage. whether or not the urine will ultimately have to be diverted to the bowel. In boys. however, replacing the bladder in the depths of the pelvis may cause a relative shortening of the urethra. so that the penis is drawn up into the abdominal wall. It is hoped that urethral lengthening will overcome this difficulty.

Lastly, one may speculate upon the effect of this operation upon the plane of the hip joints and thereby upon the gait. Any fear on this score does not seem well grounded. for three of our patients are now walking in a manner normal for their age.

\section{SUMMARY}

1. We have described an operation the aim of which is to reduce the gap at the pubic symphysis which accompanies ectopia vesicae.

2. The operation, as it is now performed, simplifies subsequent bladder closure and repair of the associated divarication of the recti.

We are grateful to $\mathrm{Mr} \mathrm{H}$. H. Nixon for his permission to report the two patients in whom he performed manual osteoclasis and to Mr Derek Martin of the Photographic Department of the Hospital for Sick Children for the illustrations.

\section{REFERENCES}

Nixon, H. H. (1957): Personal communication.

Schultz, W. G. (1958): Plastic Repair of Exstrophy of Bladder Combined with Bilateral Osteotomy of Ilia. Journal of Urology, 79, 453.

VOL. 41 B, NO. 4, NOVEMBER 1959 